\title{
The Demonstration Model of an Electromagnetic Accelerator Gun
}

\author{
Andras MOLNAR \\ John von Neumann Faculty of Informatics, Óbuda University, Budapest, \\ e-mail: molnar@uni-obuda.hu
}

Manuscript received September 19, 2018; revised December 07, 2018.

\begin{abstract}
These days a lot can be heard about special weapons which accelerate the projectile not based on the traditional, chemical energy release, but providing the muzzle velocity with the help of electromagnets. In English terminology, many descriptions can be read about these devices, referred to as "coilgun". There are so many hobbyist and amateurs who make these devices [1,2] and publish their results on the internet $[3,4]$.

The purpose of the project is dual. On one hand, features, advantages, disadvantages and the limits of the electromagnetically accelerated weapons can be found by building an experimental tool. On the other hand, it was intended to point out the fact that anybody can build such a tool using commercially available commercial components.

Although the muzzle energy of the device presented in this paper is not more than $6.8 \mathrm{~J}$, but it can cause serious injury. The paper also points out that in a similar way, still not using special components, a weapon can be made with a larger (10-20J) muzzle energy.
\end{abstract}

Keywords: Coilgun, Electromagnetic accelerator

\section{The theoretical structure of the device}

In principle, an electromagnetic accelerator is an extremely simple construction (Fig. 1). The magnetizable projectile is attracted by a magnetic field created around the electrified coil. The trajectory of the projectile is ensured by a thin-walled barrel.

The electric current flowing through the coil must be timed in such manner that there must not be a magnetic field around the coil during the exit of the projectile which would slow it down. The magnitude of the magnetic induction created by the coil is proportional to the intensity of the electric current flowing through the coil (1), which ultimately determines the accelerating force affecting the projectile. In order to calculate the magnetic induction " $\mathrm{B}$ " generated by the 
coil (solenoid) the following relation can be applied based on the 4th laws of Maxwell and Biot-Savart law (1).

Therefore, by increasing the number of turns as well as by reducing the length of the coil, magnetic induction is increased, but the creation of a short impulse demands the modification of these parameters in the opposite direction, in other words a decrease. Accordingly, the basic way to increase the magnetic induction is to increase the current through the coil. (1) The formula is correct for solenoid only, but it is enough to the analyze the effects of individual members.

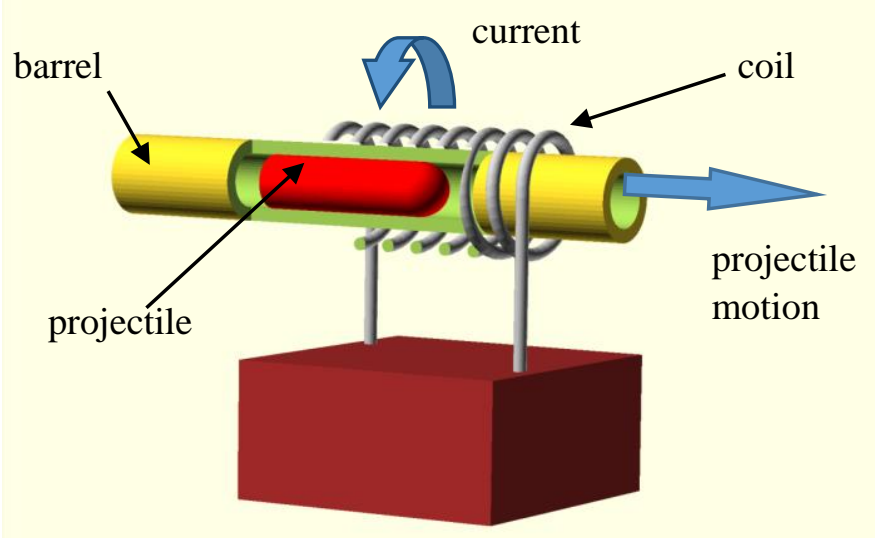

Figure 1: The structure of an electromagnetic accelerator

$$
B=\mu_{0} \frac{I * N}{l}
$$

where

$B$ is the magnitude of the magnetic field's induction generated by the coil [T] $I$ is the intensity of the current flowing in the coil [A]

$N$ is the number of turns in the coil [1]

$l$ is the length of the coil [m]

$\mu_{0}$ is the magnetic constant $\left[4 \pi * 10^{-7} \frac{\mathrm{H}}{\mathrm{m}}\right]$

In order for an even higher current to flow through the coil, firstly, it must be powered by a power source which has very low internal resistance. The most suitable option for this is the capacitor. Accordingly, the launcher is composed of a coil and a capacitor connected in parallel. In order to achieve a correct synchronization of the current impulse, a fast switching device (such is fast thyristor or spark switch [5]) must be used to connect the charged capacitor to the coil. 
In this case, the length of the impulse generated during the discharge depends on capacity and inductance values (from a practical standpoint the ohmic resistance of the closed circuit must also be taken into consideration). For the sake of a fast discharge it is advisable to use some type of a semiconductor switching device instead of a mechanical switching device. Thyristor is rather widely utilized for this purpose. At dimensioning, it must be taken into consideration that during the discharge, though only for a few milliseconds, thousands (!) of amperes flow through the coil.
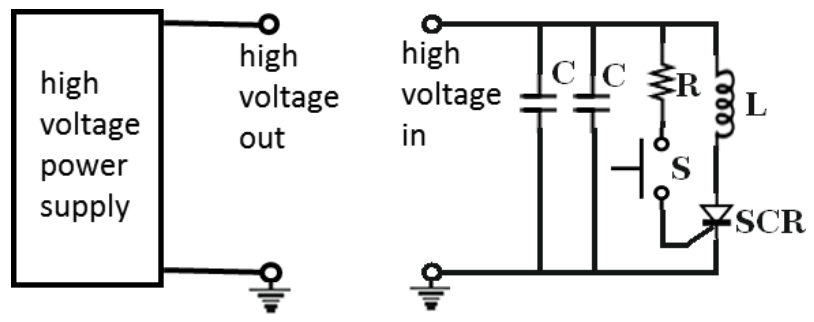

Figure 2: The theoretical circuit diagram of the electromagnetic accelerator.

The operation of the accelerator shown in Fig. 2 is the following. The "C" capacitors are charged to working voltage using an external power source. The maximum voltage of the charging power supply affects charging time only, otherwise it has no influence on the performance of the launcher. The thyristor marked "SCR" does not conduct during recharging, thus current does not flow through the work coil marked "L". The charged capacitors discharge through the coil by pushing the snap-switch marked "S".

This is the moment when firing takes place. Resistance " $R$ " provides the necessary starting voltage knowing the required voltage of the applied thyristor and the capacitors. When choosing the type of the thyristor, short current impulses must be taken into consideration, which in this current case can even be thousands of amps. Thyristors can typically even bear an impulse current larger by an order of magnitude compared to the statically permissible maximum current.

If the accelerator contains multiple stages, those have a structure similar to Fig. 2 but thyristor firing is not managed by a push-button but by the signal of some type of sensor. The easiest method to start the stage is to use an optical transmitter. When the projectile crosses the light of the transmitter, the photosensor goes into "shadow".

This signal must be connected to the controlling electrode of the switch, which starts the stage connected to it. At the scaling of further stages, the velocity of the projectile approaching the coil must be taken into consideration. Generally speaking, the faster the projectile entering the coil is, the shorter the impulse 
needs to be for acceleration. In order for acceleration to be effective the short impulse (in case the same electric power and same voltage) must be accompanied by a higher current (2).

$$
W=P * t=U * I * t
$$

where
$\mathrm{W}$ : work $[\mathrm{J}]$
P: power $[\mathrm{W}]$
$\mathrm{U}$ : voltage $[\mathrm{V}]$
I: current $[\mathrm{A}]$
t: time $[\mathrm{s}]$

The design of the three-staged electromagnetic accelerator can be seen in Fig. 3 . The first stage can be started using a switch as it was introduced previously. As a result, the projectile accelerates and proceeds inside the barrel towards the second stage. The barrel was drilled before the second stage for the light of the light source (marked with a red LED in the figure) placed below to reach the light sensor placed above it (a photo diode marked with blue color in the figure) through the two drilled holes.

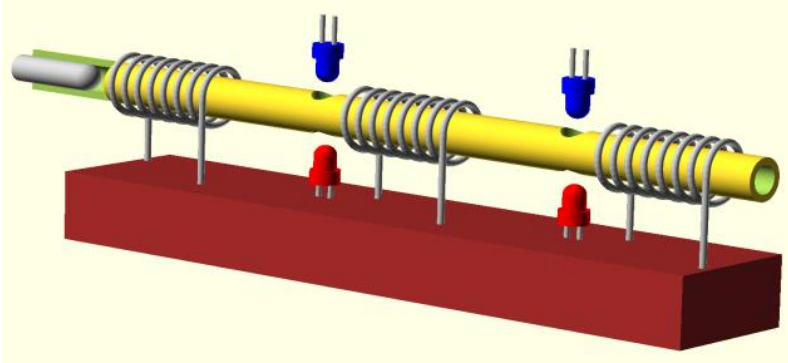

Figure 3: The design of the three-staged electronic accelerator.

The electronic impulse generated by the interruption of the way of light starts the stage connected to it which further accelerates the projectile. The distance of the optical transmitter from the coil controlled by it is a relevant parameter. When determining the distance, the switching moment of the circuit and its entry speed must be taken into consideration. In case if a too large distance, the magnetic field generated by the coil does not have a sufficient accelerating force of a substantial degree on the projectile. In case of a too short distance, it may occur that the electric current is still flowing through the coil when the projectile moves away from it, and the not yet vanished magnetic field will have a braking effect on it. The operation of the third stage is similar to the operation of the second stage that we have just shown. 
Fig. 4 demonstrates the three-staged accelerator. The three stage are coupled by the projectile. The system is practically composed of three individual units. The only common point of the units is their ground which ensures the simpler connection of the measurement devices connected to it (not visible in the figure). Every unit has its own capacitor battery and the units charging them individually (not visible in the figure).

During practical implementation, the charging circuits of the flashes, utilized for photography, were used to charge the capacitors. These circuits are designed in such a way that they are protected from the transient effects of the discharge following the charging of the capacitor connected to them, thus during their use no special attention must be paid to this.

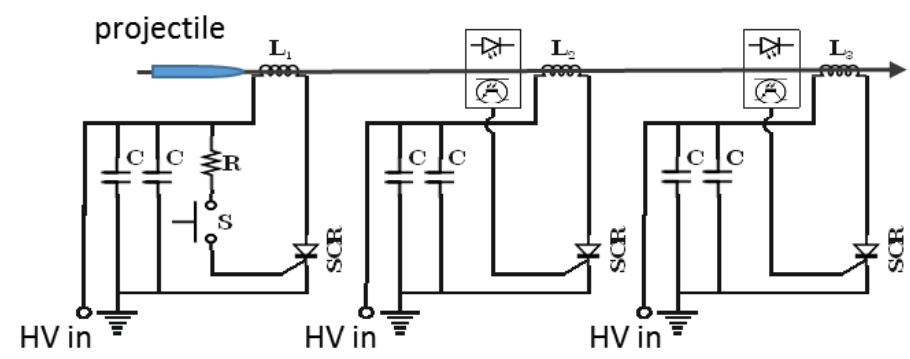

Figure 4: The theoretical circuit diagram of the three-staged electromagnetic accelerator.

\section{The electrodynamic and kinetic simulation of the device}

The acceleration of the projectile depends on several factors. In order to produce a relatively efficient device it is advisable to conduct calculations for the sake of determining the parameters of the energy banks (capacitors), the accelerating electromagnets (coils), the voltage supply, the size of the projectile and other devices. As the calculations are rather complex and the simultaneous effect of several variants influence the end result it is advisable to employ a simulation tool. During the project, the design of the device to be realized was made on the basis of the results of a program available free of charge [6]. The simulator has several "classical" calculation modules and a special kinetic module.

For the sake of facilitating handling it is sufficient to enter the main physical dimensions of the coil which the program uses to calculate the number of wire layers, the wire length to be used, the ohmic resistance and inductance. The second module conducts an LRC circuit simulation from the data from received the previous module, as well as from the value of the set capacity and from the 
value of the electric current, which shows voltage and current flow through the coil for a few milliseconds after switch-on (the time range of the simulation depends on user settings).
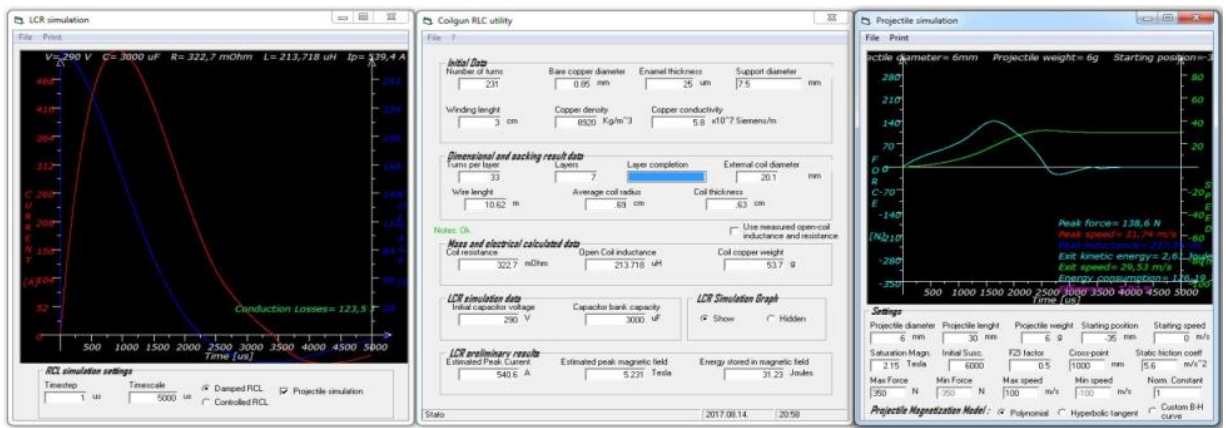

Figure 5: The simulation result of stage I.

Fig. 5 shows the simulation results of the first stage. The parameters of the simulated first stage are the following (Table 1).

Table 1: Initial parameters of the first stage

\begin{tabular}{|l|l|}
\hline coil length: & $30 \mathrm{~mm}$, \\
\hline internal diameter of the coil: & $7,5 \mathrm{~mm}$, \\
\hline number of turns in the coil: & 231, \\
\hline wire diameter: & $0,85 \mathrm{~mm}$, \\
\hline capacitor capacity: & $3000 \mu \mathrm{F}$, \\
\hline working voltage: & $290 \mathrm{~V}$ \\
\hline projectile length: & $30 \mathrm{~mm}$, \\
\hline projectile diameter: & $6,5 \mathrm{~mm}$ \\
\hline projectile mass: & $6 \mathrm{~g}$ \\
\hline starting velocity of the projectile: & $0 \mathrm{~m} / \mathrm{s}$ \\
\hline
\end{tabular}

The third larger module of the simulator software calculates and displays (Fig. 5 right-side screen shot) the motion data of the projectile. In this module it is possible to enter the initial velocity of the projectile, in this way simulating the kinematics of the second and the third stage. On the website of the software developer it can be read that the simulation contains various simplifications and conditions. Based on the tests of the developers the simulation results of the software closely approximate the results given by professional calculation models $[7,8]$, in this way the simulator, based on its recommendation, is not accurate but it provides good help during the design of an electromagnetic accelerator gun for hobby purposes. 
The current- and voltage-time diagram of the coil of stage I can be seen on the left side of Fig. 5. The left-side figure displays the velocity of the projectile in relation to time. The muzzle velocity of the projectile can be read from the figure (as the program conducts only a one-staged simulation). In the present case this value is around $27 \mathrm{~m} / \mathrm{s}$. This velocity value will be the initial velocity of the projectile at the simulation of the next stage.

It is worth mentioning that in case of leaving all parameters unchanged the accelerator ensures only $27 \mathrm{~m} / \mathrm{s}$ exit velocity in case of a projectile with $0 \mathrm{~m} / \mathrm{s}$ starting speed. If the second stage matches the first one in every parameter, it will slow down the projectile arriving at $27 \mathrm{~m} / \mathrm{s}$ speed instead of accelerating it (Fig. 6). It can be seen in Fig. 6 that the entry speed of the projectile is $29 \mathrm{~m} / \mathrm{s}$, and at the exit of this the stage it is only $23 \mathrm{~m} / \mathrm{s}$.

The reason for this is that the discharge time of stage I is too long, and in this way the magnetic field "pulls back" the projectile. Discharge time can be reduced if the capacity of the capacitor bank is reduced, but then the energy released during the discharge will be smaller. This is the reason why it is more advisable to reduce its inductance by reducing the turn number of the coil. Another effect of reducing the turn number is that discharge current will increase, thus when choosing the thyristor suitable for the stage this must be taken into consideration.

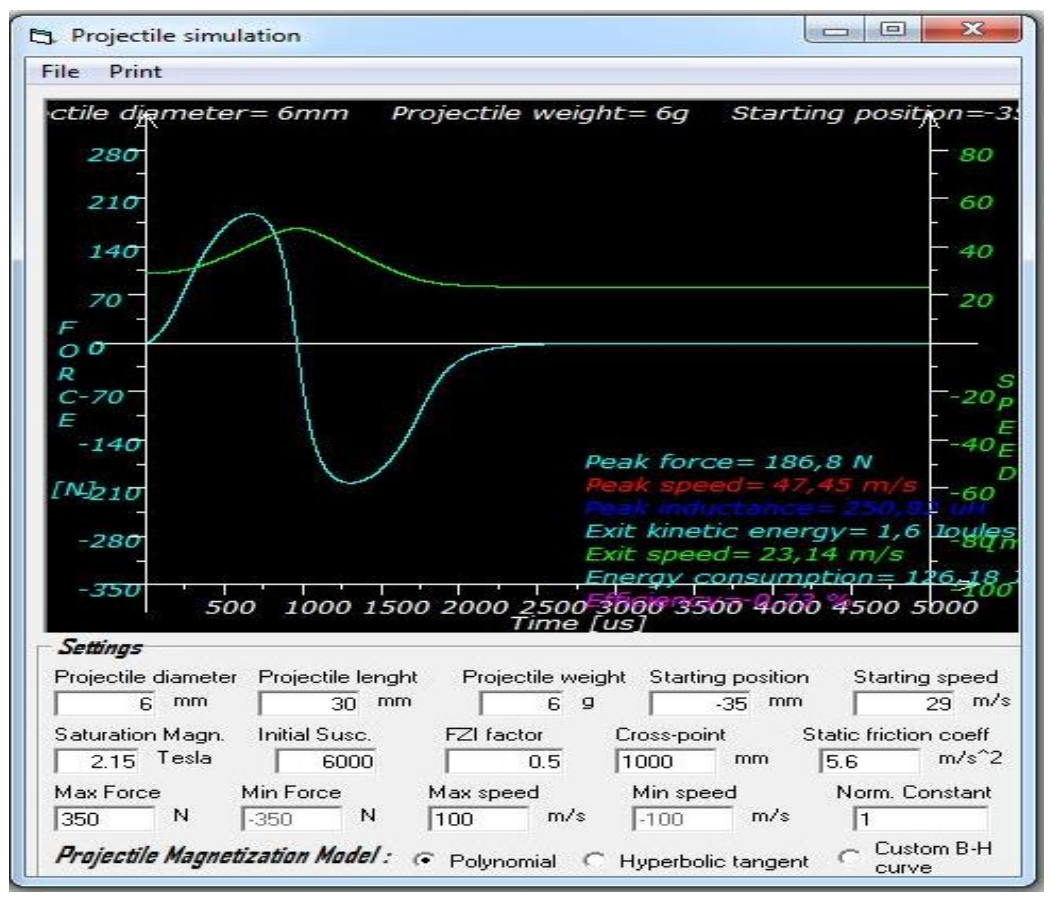

Figure 6: The slowing effect of stage I in case of the projectile with $29 \mathrm{~m} / \mathrm{s}$ entry speed. 


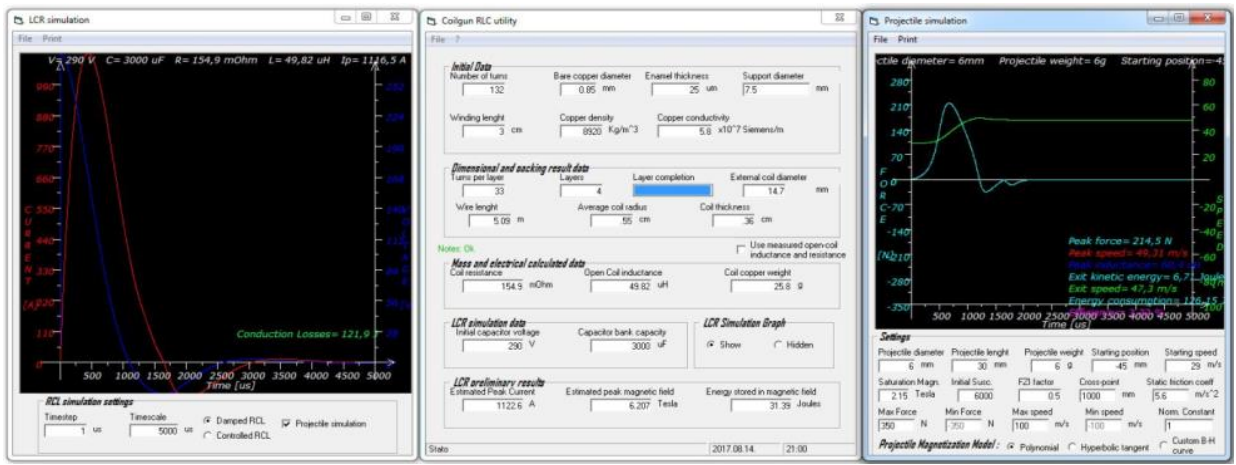

Figure 7: The simulation result of stage II.

Fig. 7 shows the simulation of stage II. The velocity of the projectile arriving to the stage is the exit velocity resulting from the previous simulation.

As the data of the projectile are obviously unchanged, except velocity, the internal diameter of the coil does not change either, these are not displayed in the following list of parameters of the second stage (Table 2).

Table 2: Parameters of the stage II.

\begin{tabular}{|l|l|}
\hline coil length: & $30 \mathrm{~mm}$, \\
\hline coil turn number: & 132, \\
\hline wire diameter: & $0,85 \mathrm{~mm}$, \\
\hline capacitor capacity: & $3000 \mu \mathrm{F}$, \\
\hline working voltage: & $290 \mathrm{~V}$ \\
\hline starting velocity of the projectile: & $29 \mathrm{~m} / \mathrm{s}$ \\
\hline
\end{tabular}

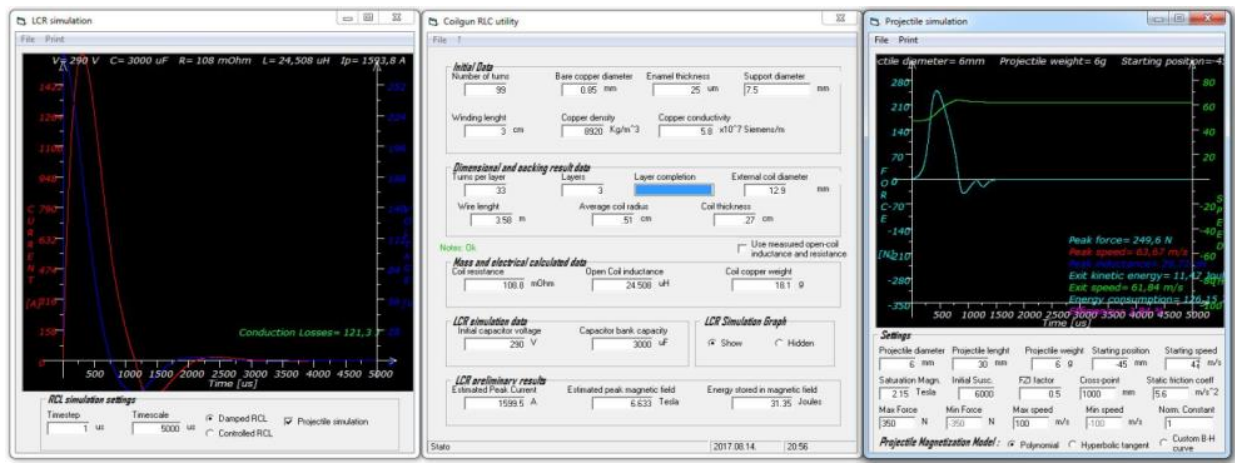

Figure 8: The simulation result of stage III. 
It can be seen from the simulation that whereas in case of the first stage the discharge peak current is $540 \mathrm{~A}$, in case of the second stage this is now $1122 \mathrm{~A}$. Leaving the stage, the velocity of the projectile reaches $47 \mathrm{~m} / \mathrm{s}$.

It can be read from Fig. 8 that the maximum velocity of the projectile is more than $61 \mathrm{~m} / \mathrm{s}$ which in case of the given projectile means more than $11 \mathrm{~J}$ muzzle energy. The parameters of stage III. are the following (Table 3).

Table 3: Initial parameters of the stage III.

\begin{tabular}{|l|l|}
\hline coil length: & $30 \mathrm{~mm}$, \\
\hline coil turn number: & 99, \\
\hline wire diameter: & $0,85 \mathrm{~mm}$, \\
\hline capacitor capacity: & $3000 \mu \mathrm{F}$, \\
\hline working voltage: & $290 \mathrm{~V}$ \\
\hline starting velocity of the projectile: & $47 \mathrm{~m} / \mathrm{s}$ \\
\hline
\end{tabular}

A frame construction is needed to build the structure determined using simulations, as the position of the coils and their transmitters must be kept accurate. During the simulation it was clearly revealed that the accelerator is very sensitive to projectile parameters, furthermore, to synchronization of the triggering of the stages with the position of the projectile. As the failure of the mechanical construction may thwart the operation of the device, it is advised to build sufficiently stable mechanics, in other words one which keeps the position of the parts stable. Sufficiently flexible, easily plannable and executable mechanics can be built by using 3D printing. Furthermore, the procedure is sufficiently economical too.

\section{Experimental calculations, experiments}

Calculations were conducted on our experimental device to observe whether the previously calculated results and the nature of the temporal changes corrolate with each other. As a first step, we examined whether the variation in time of the coil voltage corresponds to the expectations. By conducting the measurements using a multi-channel oscilloscope with digital storage, we received the oscillogram corresponding to Fig. 14.

The minimal deviations of the initial DC voltages, seen in the figure, can be experienced due to the slightly differing settings of the independent charging circuits in certain coils. It is clearly visible that stage by stage the voltage slopes become steeper and steeper (Fig. 9).

The results of the kinematic measurements are demonstrated by Fig. 9 and Fig. 10. The first measurements are directed towards the question whether the given stages indeed further accelerate the projectile. In case of improper settings, indeed, it occurs often that further stages, because of the wrong timing, pull back 
the projectile accelerated by the previous stage and as a result the multi-staged device is weaker than the accelerator composed of one stage.

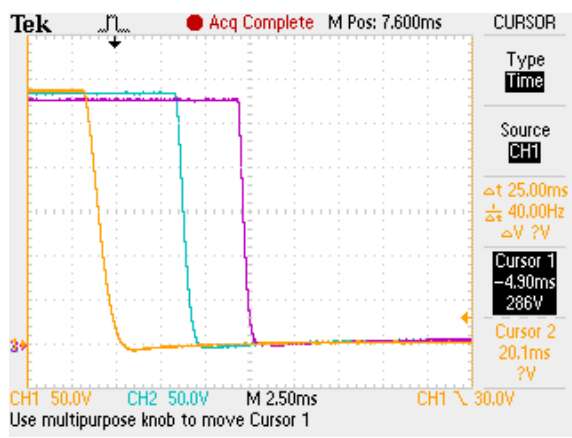

Figure 9: Oscillograms of the three coil voltages during a shot.

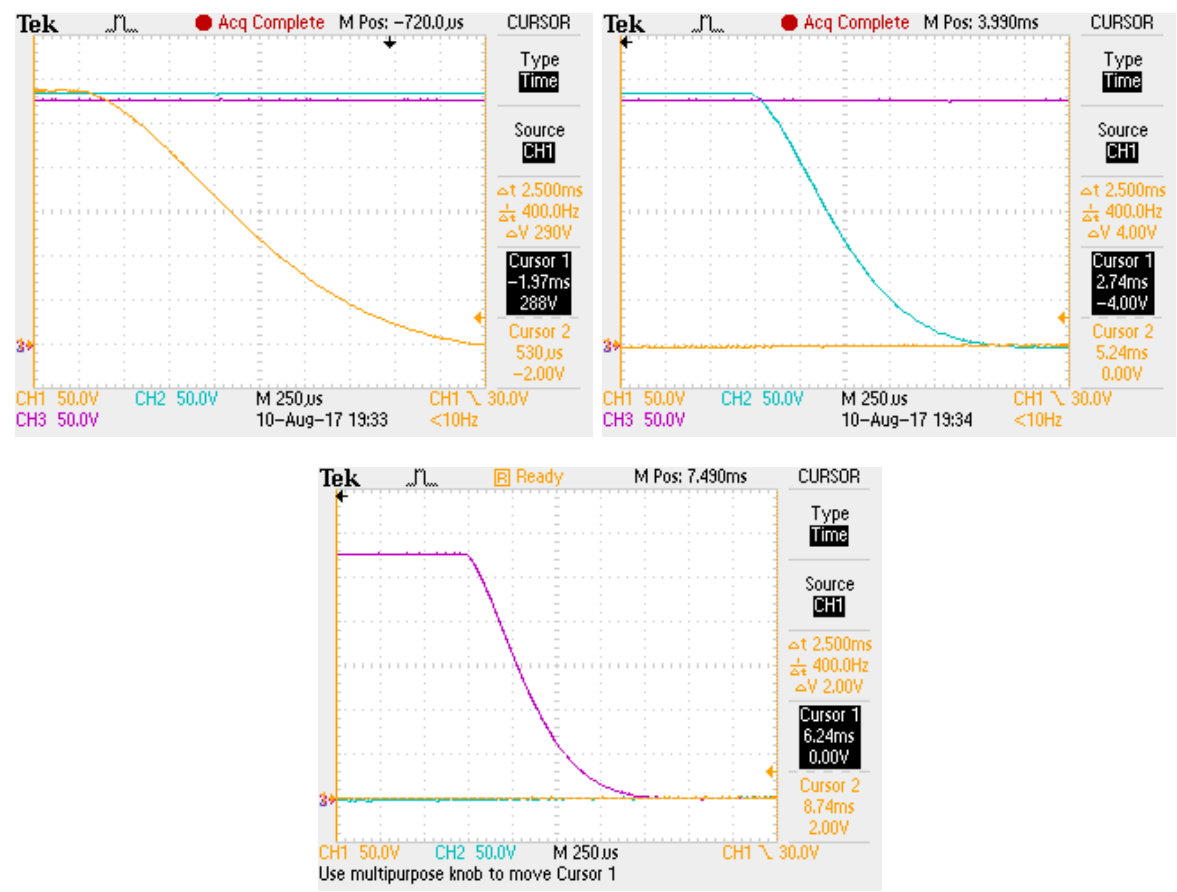

Figure 10: Oscillograms of individual stages during a shot.

Table 4 demonstrates the effect of the given stages. It is clearly visible that both the second and the third stages increase the projectile's velocity. The three stages together resulted in $4.5 \mathrm{~J}$ muzzle energy during the experiment which was below simulation results (a value around $11 \mathrm{~J}$ ). Its reason may come from several 
sources. The deviation is partly caused by the inaccurate design of the coils and largely by the timing of the coil magnetization.

Table 4: The variation of muzzle velocity depending on the number of accelerating stages

\begin{tabular}{|l|c|c|c|}
\hline & Stage I. & Stage I. + II. & Stage I. + II. + III. \\
\hline $\begin{array}{l}\text { 1st measurement } \\
\text { muzzle velocity [m/s] }\end{array}$ & 23,39 & 34,2 & 39,3 \\
\hline $\begin{array}{l}\text { 2nd measurement } \\
\text { muzzle velocity [m/s] }\end{array}$ & 23,51 & 33,9 & 39,6 \\
\hline $\begin{array}{l}\text { 3rd measurement muzzle } \\
\text { velocity[m/s] }\end{array}$ & 22,28 & 35,2 & 38,4 \\
\hline \hline average muzzle velocity [m/s] & 23,06 & 34,43 & 39,1 \\
\hline \hline average muzzle energy [m/s] & $\sim 1,6$ & $\sim 3,5$ & $\sim 4,6$ \\
\hline
\end{tabular}

Table 5: The variant of muzzle energy depending on the mass of the projectile and its starting position

\begin{tabular}{|l|c|c|c|c|}
\hline & \multicolumn{2}{|c|}{6 gram projectile } & \multicolumn{2}{c|}{5 gram projectile } \\
\cline { 2 - 5 } & $\begin{array}{c}0 \mathrm{~mm} \\
\text { starting } \\
\text { position }\end{array}$ & $\begin{array}{c}-2 \mathrm{~mm} \\
\text { starting } \\
\text { position }\end{array}$ & $\begin{array}{c}0 \mathrm{~mm} \\
\text { starting } \\
\text { position }\end{array}$ & $\begin{array}{c}-2 \mathrm{~mm} \\
\text { starting } \\
\text { position }\end{array}$ \\
\hline $\begin{array}{l}\text { 1st measurement } \\
\text { muzzle velocity [m/s] }\end{array}$ & 39,3 & 47,0 & 48,2 & 52,5 \\
\hline $\begin{array}{l}\text { 2nd measurement } \\
\text { muzzle velocity [m/s] }\end{array}$ & 39,6 & 45,4 & 48,4 & 52,4 \\
\hline $\begin{array}{l}\text { 3rd measurement } \\
\text { muzzle velocity [m/s] }\end{array}$ & 38,4 & 45,5 & 48,5 & 52,4 \\
\hline \hline $\begin{array}{l}\text { average muzzle } \\
\text { velocity [m/s] }\end{array}$ & 39,1 & 45,96 & 48,36 & 52,43 \\
\hline \hline $\begin{array}{l}\text { average muzzle } \\
\text { energy [J] }\end{array}$ & $\sim 4,6$ & $\sim 6,3$ & $\sim 5,8$ & 6,8 \\
\hline
\end{tabular}

As the initial positon of the projectile is crucial regarding performance, measurements have been conducted with different starting positions. Apart from the use of all three accelerator coils, Table 5 demonstrates the effect of the initial position and the effect of the used projectile's mass.

Reducing the mass of the projectile resulted in a larger muzzle energy which occurred due to the improvement of the acceleration's performance. The same projectile slightly pushed inwards into the barrel gathered significant extra energy compared to the one placed at the end of the tube. During several experiments, the muzzle energy of the projectile placed more inwards from the end of the tube proved to be the largest. In this way, using the projectile with $5 \mathrm{~g}$ mass $6.8 \mathrm{~J}$ 
muzzle energy was achieved which is still far behind theoretical, calculated values.

\section{Conclusion}

As the synchronization time of the coils' magnetization with the position of the projectile is crucial, the theoretical energy level may be approximated by correct triggering of stages II. and III. The device however is not suitable for these stages to be set. In this way it is necessary to build another experimental device to achieve maximum muzzle energy [9]. In this prototype the optical trigger unit is firmly fixed to the barrel. For the new design, the optical trigger unit position must be movable mounted to fine-tune the optimum magnetization moment. In the new gun, measuring the velocity of the projectile must be done before each unit to determine the optimal magnetization time of the coil. In the absence of the speed measurement units in the prototype, the coil magnetization time optimization is not possible.

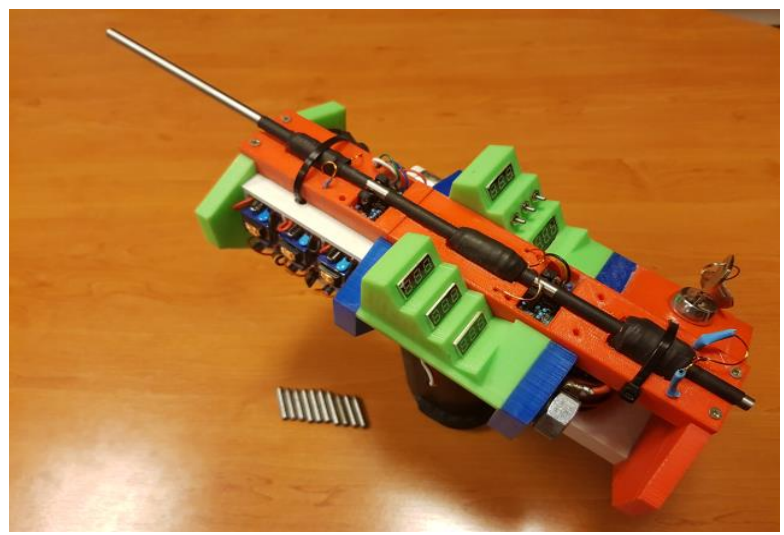

Figure 11: Photo of the created device.

\section{References}

[1] Burgess, T. J., Cnare, E. C., W. L. Oberkampf, Beard, S. G., and Cowan, M. "The Electromagnetic Theta Gun and Tubular Projectiles", IEEE Trans. on Magnetics, vol. mag-18, no. 1, Jan. 1982.

[2] Andrews and, J. A., Devine, J. R. “Armature Design for Coaxial Induction Launchers”, IEEE Trans. on Magnetics, vol. 27, no. 1, Jan. 1991.

[3] http://www.coilgun.eclipse.co.uk/

[4] https://www.coilgun.info/

[5] Kharlov, A. V. "Numerical simulation of spark channel dynamics in railgun switches" Journal of Physics Conference Series 1115:022027, Nov. 2018 
[6] http://www.webalice.it/saverio.filippi/coilgun_simulator.html

[7] He, Y. et al. "Efficiency analysis of an electromagnetic railgun with a full circuit model" IEEE Trans. Plasma Sci., vol. 38, no. 12, pp. 3425-3428, Dec. 2010

[8] Kulkarni, A. S., Thomas, M. J. "Design of a Compulsator to Drive a Railgun" IEEE Transactions on Plasma Science, 2010, vol. 45 no.7, pp. 1482-1488

[9] Engel, T. G., Neri, J. M., and Veracka, M. J. "The maximum theoretical efficiency of constant inductance gradient electromagnetic launchers" IEEE Trans. Plasma Sci., vol. 37, no. 4, pp. 608-614, Apr. 2009 